\title{
Solutions and Conservation Laws of a (2+1)-Dimensional Boussinesq Equation
}

\author{
Letlhogonolo Daddy Moleleki and Chaudry Masood Khalique \\ International Institute for Symmetry Analysis and Mathematical Modelling, Department of Mathematical Sciences, \\ North-West University, Mafikeng Campus, Private Bag X 2046, Mmabatho 2735, South Africa
}

Correspondence should be addressed to Chaudry Masood Khalique; masood.khalique@nwu.ac.za

Received 6 July 2013; Accepted 19 August 2013

Academic Editor: Maria Gandarias

Copyright (C) 2013 L. D. Moleleki and C. M. Khalique. This is an open access article distributed under the Creative Commons Attribution License, which permits unrestricted use, distribution, and reproduction in any medium, provided the original work is properly cited.

\begin{abstract}
We study a nonlinear evolution partial differential equation, namely, the $(2+1)$-dimensional Boussinesq equation. For the first time Lie symmetry method together with simplest equation method is used to find the exact solutions of the $(2+1)$-dimensional Boussinesq equation. Furthermore, the new conservation theorem due to Ibragimov will be utilized to construct the conservation laws of the (2+1)-dimensional Boussinesq equation.
\end{abstract}

\section{Introduction}

Nonlinear evolution equations (NLEEs) are broadly used as models to represent physical phenomena in numerous fields of sciences, especially in biology, solid state physics, plasma physics, plasma waves, and fluid mechanics. It is therefore of paramount importance that exact solutions of such NLEEs are obtained. However, finding exact solutions of NLEEs is an onerous exercise and only in certain distinctive cases one can explicitly write down their solutions. Nevertheless, in the last few decades important progress has been made and many powerful and effective methods for obtaining exact solutions of NLEEs have been suggested in the literature. Some of the important methods found in the literature include the Darboux transformation method [1], the inverse scattering transform method [2], Hirota’s bilinear method [3], Jacobi elliptic function expansion method [4], the sine-cosine method [5], the auxiliary ordinary differential equation method [6], Lie symmetry analysis [7-11], the F-expansion method [12], and the exp-function expansion method [13].

In this paper we consider one such NLEE, namely, the $(2+1)$-dimensional Boussinesq equation given by

$$
u_{t t}-u_{x x}-u_{y y}-\alpha\left(u^{2}\right)_{x x}-u_{x x x x}=0
$$

which describes the propagation of gravity waves on the surface of water; in particular it describes the head-on collision of an oblique wave. The (2+1)-dimensional Boussinesq equation (1) combines the two-way propagation of the classical Boussinesq equation with the dependence on a second spatial variable, as that occurs in the two-dimensional Kadomstev-Petviashvili (KP) equation. This equation provides a description of head-on collision of oblique waves and it possesses some interesting properties. The unknown function describes the elevation of the free surface of the fluid. Moreover, (1) involves the two dissipative terms $u_{x x}$ and $u_{y y}$ in addition to the fourth-order spatial derivative $u_{x x x x}$ that represents the dispersion phenomenon. Unlike the standard Boussinesq equation, which is completely integrable, that admits multiple solitons solutions, (1) is not integrable and gives two soliton solutions at most. It is to be noted that if we delete the dissipative term $u_{y y}$ from (1), we obtain the standard Boussinesq equation. The standard Boussinesq equation arises in many physical applications such as nonlinear lattice waves and iron sound waves in plasma and in vibrations in a nonlinear string. It is used in many physical applications such as the percolation of water in porous subsurface of a horizontal layer of material. See also [14].

In [15] the authors used a generalized transformation in homogeneous balance method and found some explicit solitary wave solutions of the (2+1)-dimensional Boussinesq equation. Applied homotopy perturbation method was used in [16] to construct numerical solutions of (1). Extended 
ansätz method was employed in [17] to derive exact periodic solitary wave solutions. Recently, the Hirota bilinear method was used in [18] to obtain two soliton solutions.

Lie group method is one of the most effective methods to find solutions of nonlinear partial differential equations (PDEs). Originally, developed by Sophus Lie (1842-1899) in the latter half of the nineteenth century, this method is based upon the study of the invariance under one parameter Lie group of point transformations and it is highly algorithmic [7-9].

In the study of PDEs, conservation laws play a vital role in their solution process and the reduction of PDEs. Conservation laws have been broadly used in studying the existence, uniqueness, and stability of solutions of nonlinear PDEs (see, e.g., [19-21]). They have also been used in the development of numerical methods (see, e.g., [22, 23]). Exact solutions (by exploiting a double reduction method) of some classical partial differential equations have been obtained using conserved vectors associated with the Lie point symmetries [24-26].

In this paper, for the first time, Lie group analysis in conjunction with the simplest equation method $[27,28]$ is employed to obtain some exact solutions of (1). In addition to this, conservation laws will be derived for (1) using the new conservation theorem due to Ibragimov [29].

\section{Solutions of (1)}

In this section we obtain exact solutions of (1) using Lie group analysis along with the simplest equation method.

2.1. Exact Solutions Using Lie Point Symmetries. In this subsection we first calculate the Lie point symmetries of (1) and later use the translation symmetries to construct the exact solutions.

2.1.1. Lie Point Symmetries. The symmetry group of the $(2+1)$ dimensional Boussinesq equation (1) will be generated by the vector field of the form

$$
R=\xi^{1} \frac{\partial}{\partial x}+\xi^{2} \frac{\partial}{\partial y}+\xi^{3} \frac{\partial}{\partial t}+\eta \frac{\partial}{\partial u},
$$

where $\xi^{i}, i=1,2,3$ and $\eta$ depend on $x, y, t$ and $u$. Applying the fourth prolongation $\mathrm{pr}^{(4)} R$ to (1) we obtain an overdetermined system of linear partial differential equations. Solving this resultant system one obtains the following five Lie point symmetries:

$$
\begin{gathered}
R_{1}=\frac{\partial}{\partial x}, \quad R_{2}=\frac{\partial}{\partial t}, \quad R_{3}=\frac{\partial}{\partial y}, \\
R_{4}=y \frac{\partial}{\partial t}+t \frac{\partial}{\partial y} \\
R_{5}=-2 \alpha t \frac{\partial}{\partial t}-\alpha x \frac{\partial}{\partial x}-2 \alpha y \frac{\partial}{\partial y}+(1+2 \alpha u) \frac{\partial}{\partial u} .
\end{gathered}
$$

We now utilize the symmetry $R=R_{1}+R_{2}+c R_{3}$, where $c$ is a constant, and reduce the Boussinesq equation (1) to a PDE in two independent variables. Solving the associated Lagrange system for $R$, we obtain the following three invariants:

$$
f=y-c t, \quad g=t-x, \quad \theta=u .
$$

Now treating $\theta$ as the new dependent variable and $f$ and $g$ as new independent variables, the Boussinesq equation (1) transforms to

$$
\left(1-c^{2}\right) \theta_{f f}+2 c \theta_{f g}+2 \alpha \theta_{g}^{2}+2 \alpha \theta \theta_{g g}+\theta_{g g g g}=0,
$$

which is a nonlinear PDE in two independent variables. We now use the Lie point symmetries of (5) and transform it to an ordinary differential equation (ODE). The equation (5) has the following three symmetries:

$$
\begin{gathered}
\Gamma_{1}=\frac{\partial}{\partial g}, \quad \Gamma_{2}=\frac{\partial}{\partial f}, \\
\Gamma_{3}=\left(2 \alpha f-2 \alpha f c^{2}\right) \frac{\partial}{\partial f}+\left(\alpha c f-c^{2} \alpha g+\alpha g\right) \frac{\partial}{\partial g} \\
+\left(c^{2}+2 c^{2} \alpha \theta-2 \alpha \theta\right) \frac{\partial}{\partial \theta} .
\end{gathered}
$$

The combination of the first two translational symmetries, $\Gamma=\Gamma_{1}+\nu \Gamma_{2}$, where $v$ is a constant, yields the two invariants

$$
z=f-v g, \quad \psi=\theta,
$$

which gives rise to a group-invariant solution $\psi=\psi(z)$, and consequently using these invariants, (5) is transformed into the fourth-order nonlinear ODE

$$
2 \alpha \nu^{2} \psi^{\prime 2}+\left(1-c^{2}-2 c \nu\right) \psi^{\prime \prime}+2 \alpha \nu^{2} \psi \psi^{\prime \prime}+\nu^{4} \psi^{\prime \prime \prime \prime}=0 .
$$

Integrating the above equation four times and taking the constants of integration to be zero (because we are looking for soliton solutions) and reverting back to the original variables, we obtain the following group-invariant solutions of the Boussinesq equation (1):

$$
u(x, y, t)=\frac{A_{1}}{A_{2}} \operatorname{sech}^{2}\left[\frac{\sqrt{A_{1}}}{2}(B \pm z)\right],
$$

where $B$ is a constant of integration and

$$
\begin{gathered}
A_{1}=\frac{c^{2}+2 v c-1}{v^{4}}, \quad A_{2}=\frac{2 \alpha}{3 v^{2}}, \\
z=v x+y-(c+v) t .
\end{gathered}
$$

It is worth noting that the obtained solitary wave solution $u(x, y, t)$ is a regular soliton in the form of a bell-shaped soliton.

2.2. Exact Solutions of (1) Using Simplest Equation Method. In this section we employ the simplest equation method [27, $28]$ to solve the nonlinear ODE (8). This will then give us the exact solutions for our Boussinesq equation (1). The simplest equations that we will use in our work are the Bernoulli and Riccati equations. 
Here we first present the simplest equation method and consider the solutions of (8) in the form

$$
F(z)=\sum_{i=0}^{M} A_{i}(G(z))^{i},
$$

where $G(z)$ satisfies the Bernoulli and Riccati equations, $M$ is a positive integer that can be determined by balancing procedure, and $A_{0}, \ldots, A_{M}$ are parameters to be determined.

The Bernoulli equation

$$
G^{\prime}(z)=a G(z)+b G^{2}(z),
$$

where $a$ and $b$ are arbitrary constants has the general solution given by

$$
G(z)=a\left\{\frac{\cosh [a(z+C)]+\sinh [a(z+C)]}{1-b \cosh [a(z+C)]-b \sinh [a(z+C)]}\right\}
$$

and represents a solitary wave solution.

The Riccati equation considered in this work is

$$
G^{\prime}(z)=b G^{2}(z)+a G(z)+d,
$$

where $a, b$, and $d$ are arbitrary constants. Its solutions are

$$
\begin{gathered}
G(z)=-\frac{a}{2 b}-\frac{\theta}{2 b} \tanh \left[\frac{1}{2} \theta(z+C)\right], \\
G(z)=-\frac{a}{2 b}-\frac{\theta}{2 b} \tanh \left(\frac{1}{2} \theta z\right) \\
+\frac{\operatorname{sech}(\theta z / 2)}{C \cosh (\theta z / 2)-(2 b / \theta) \sinh (\theta z / 2)},
\end{gathered}
$$

with $\theta^{2}=a^{2}-4 b d$ and $C$ is an arbitrary constant of integration.

2.2.1. Solutions of (1) Using the Bernoulli Equation as the Simplest Equation. The balancing procedure gives $M=2$ so the solutions of (8) are of the form

$$
F(z)=A_{0}+A_{1} G+A_{2} G^{2} .
$$

Inserting (16) into (8) and using the Bernoulli equation (12) and, thereafter, equating the coefficients of powers of $G^{i}$ to zero, we obtain an algebraic system of six equations in terms of $A_{0}, A_{1}$, and $A_{2}$, namely,

$$
\begin{gathered}
-120 v^{4} A_{2} b^{4}-20 \alpha v^{2} A_{2}^{2} b^{2}=0, \\
-336 v^{4} A_{2} a b^{3}-36 \alpha v^{2} A_{2}^{2} a b-24 v^{4} A_{1} b^{4} \\
-24 \alpha v^{2} A_{1} A_{2} b^{2}=0, \\
-A_{1} a^{2}+2 v A_{1} a^{2} c-v A_{1} a^{4}+A_{1} a^{2} c^{2} \\
-2 \alpha \nu^{2} A_{1} A_{2} a^{2}=0, \\
-16 \alpha \nu^{2} A_{2}^{2} a^{2}+12 v A_{2} b^{2} c-6 \alpha \nu^{2} A_{1}^{2} b^{2} \\
-60 v^{4} A_{1} a b^{3}-12 \alpha \nu^{2} A_{0} A_{1} b^{2}-330 v^{4} A_{2} a^{2} b^{2} \\
-42 \alpha \nu^{2} A_{1} a b A_{2}+6 A_{2} b^{2} c^{2}-6 A_{2} b^{2}=0,
\end{gathered}
$$

$$
\begin{aligned}
- & 15 v^{4} A_{1} a^{3} b+8 v A_{2} a^{2} c-3 A_{1} a b-4 A_{2} a^{2} \\
+ & 4 A_{2} a^{2} c^{2}-4 \alpha v^{2} A_{1}^{2} a^{2}-6 \alpha v^{2} A_{0} A_{1} a b-16 v^{4} A_{2} a^{4} \\
+ & 6 A_{1} a b c v-8 \alpha v^{2} A_{0} A_{2} a^{2}+3 A_{1} a b c^{2}=0, \\
- & 18 \alpha v^{2} A_{1} A_{2} a^{2}-10 \alpha v^{2} A_{1}^{2} a b-4 \alpha v^{2} A_{0} A_{1} b^{2} \\
& +10 A_{2} a b c^{2}+4 v A_{1} b^{2} c+20 v A_{2} a b c-2 A_{1} b^{2} \\
& +2 A_{1} b^{2} c^{2}-20 \alpha v^{2} A_{0} A_{2} a b-10 A_{2} a b \\
& -130 v^{4} A_{2} a^{3} b-50 v^{4} A_{1} a^{2} b^{2}=0 .
\end{aligned}
$$

With the aid of Mathematica, solving the above system of algebraic equations, one possible solution for $A_{0}, A_{1}$, and $A_{2}$ is

$$
\begin{gathered}
A_{0}=\frac{-\left(1-c^{2}-2 c v+a^{2} v^{4}\right)}{2 \alpha v^{2}}, \\
A_{1}=\frac{-6 a b v^{2}}{\alpha}, \\
A_{2}=\frac{-6 b^{2} v^{2}}{\alpha} .
\end{gathered}
$$

Thus, reverting back to the original variables, a solution of (1) is

$$
\begin{aligned}
u(t, x, y) & \\
= & A_{0}+A_{1} a\left\{\frac{\cosh [a(z+C)]+\sinh [a(z+C)]}{1-b \cosh [a(z+C)]-b \sinh [a(z+C)]}\right\} \\
& +A_{2} a^{2}\left\{\frac{\cosh [a(z+C)]+\sinh [a(z+C)]}{1-b \cosh [a(z+C)]-b \sinh [a(z+C)]}\right\}^{2},
\end{aligned}
$$

where $z=v x+y-(c+v) t$ and $C$ is an arbitrary constant of integration, which represents a solitary wave solution.

\subsubsection{Solutions of (1) Using the Riccati Equation as the Simplest} Equation. The balancing procedure yields $M=2$ so the solutions of (8) take the form

$$
F(z)=A_{0}+A_{1} G+A_{2} G^{2} .
$$

Inserting (20) into (8) and making use of the Riccati equation (14), we obtain algebraic system of equations in terms of $A_{0}$, $A_{1}$, and $A_{2}$ by equating the coefficients of powers of $G^{i}$ to zero. The resulting algebraic equations are

$$
\begin{gathered}
-120 \nu^{4} A_{2} b^{4}-20 \alpha v^{2} A_{2}^{2} b^{2}=0, \\
-36 \alpha v^{2} A_{2}^{2} a b-336 v^{4} A_{2} a b^{3}-24 v^{4} A_{1} b^{4} \\
-24 \alpha \nu^{2} A_{1} A_{2} b^{2}=0,
\end{gathered}
$$




$$
\begin{aligned}
& -32 \alpha v^{2} A_{2}^{2} b d-6 \alpha v^{2} A_{1}^{2} b^{2}-240 v^{4} A_{2} b^{3} d \\
& +6 A_{2} b^{2} c^{2}+12 A_{2} b^{2} c \nu-6 A_{2} b^{2} \\
& -12 \alpha \nu^{2} A_{0} A_{1} b^{2}-42 \alpha \nu^{2} A_{1} A_{2} a b-16 \alpha v^{2} A_{2}^{2} a^{2} \\
& -60 v^{4} A_{1} a b^{3}-330 A_{2} a^{2} b^{2}=0, \\
& -16 v^{4} A_{2} b d^{3}-14 v^{4} A_{2} a^{2} d^{2}+2 A_{1} a c d v \\
& -A_{1} a d+4 A_{2} c d^{2} v+A_{1} a c^{2} d-8 v^{4} A_{1} a b d^{2} \\
& +2 A_{2} c^{2} d^{2}-v^{4} A_{1} a^{3} d-2 \alpha v^{2} A_{0} A_{1} a d \\
& -4 \alpha \nu^{2} A_{0} A_{2} d^{2}-2 \alpha \nu^{2} A_{1}^{2} d^{2}-2 A_{2} d^{2}=0, \\
& 2 A_{1} b^{2} c^{2}-28 \alpha v^{2} A_{2}^{2} a d-20 \alpha v^{2} A_{0} A_{2} a b \\
& -36 \alpha v^{2} A_{1} A_{2} b d-18 \alpha \nu^{2} A_{1} A_{2} a^{2}-10 \alpha v^{2} A_{1}^{2} a b \\
& -10 A_{2} a b+10 A_{2} a b c^{2}+4 A_{1} b^{2} c v-40 v^{4} A_{1} b^{3} d \\
& -4 \alpha v^{2} A_{0} A_{1} b^{2}-50 v^{4} A_{1} a^{2} b^{2}+20 A_{2} a b c v-2 A_{1} b^{2} \\
& -130 v^{4} A_{2} a^{3} b-440 v^{4} A_{2} a b^{2} d=0, \\
& 2 A_{1} a^{2} c v-6 A_{2} a d-v^{4} A_{1} a^{4} \\
& +12 A_{2} a c d v-6 \alpha v^{2} A_{1}^{2} a d+6 A_{2} a c^{2} d \\
& +A_{1} a^{2} c^{2}-12 \alpha v^{2} A_{1} A_{2} d^{2}-4 \alpha v^{2} A_{0} A_{1} b d \\
& -120 v^{4} A_{2} a b d^{2}+4 A_{1} b c d v-2 \alpha v^{2} A_{0} A_{1} a^{2} \\
& -12 \alpha v^{2} A_{0} A_{2} a d-16 v^{4} A_{1} b^{2} d^{2}-A_{1} a^{2} \\
& -30 v^{4} A_{2} a^{3} d-2 A_{1} b d+2 A_{1} b c^{2} d \\
& -22 v^{4} A_{1} a^{2} b d=0, \\
& -8 \alpha v^{2} A_{0} A_{2} a^{2}+3 A_{1} a b c^{2}-8 A_{2} b d \\
& +6 A_{1} a b c v-3 A_{1} a b-6 \alpha v^{2} A_{0} A_{1} a b \\
& -136 v^{4} A_{2} b^{2} d^{2}-4 A_{2} a^{2}-12 \alpha v^{2} A_{2}^{2} d^{2} \\
& +8 A_{2} a^{2} c v-16 \alpha v^{2} A_{0} A_{2} b d-232 v^{4} A_{2} a^{2} b d \\
& -8 \alpha v^{2} A_{1}^{2} b d+16 A_{2} b c d v-15 v^{4} A_{1} a^{3} b \\
& -16 v^{4} A_{2} a^{4}-60 v^{4} A_{1} a b^{2} d+8 A_{2} b c^{2} d \\
& +4 A_{2} a^{2} c^{2}-30 \alpha v^{2} A_{1} A_{2} a d-4 \alpha v^{2} A_{1}^{2} a^{2}=0 .
\end{aligned}
$$

Solving the above equations, we get

$$
\begin{aligned}
& A_{0}=\frac{-8 b d \nu^{4}-a^{2} v^{4}+c^{2}+2 c \nu-1}{2 v^{2} \alpha}, \\
& A_{1}=\frac{-6 a b v^{2}}{\alpha}, \quad A_{2}=\frac{-6 b^{2} v^{2}}{\alpha},
\end{aligned}
$$

and consequently, the solutions of (1) are

$$
\begin{aligned}
& u(t, x, y) \\
& =A_{0}+A_{1}\left\{-\frac{a}{2 b}-\frac{\theta}{2 b} \tanh \left[\frac{1}{2} \theta(z+C)\right]\right\} \\
& \quad+A_{2}\left\{-\frac{a}{2 b}-\frac{\theta}{2 b} \tanh \left[\frac{1}{2} \theta(z+C)\right]\right\}^{2}, \\
& u(t, x, y) \\
& =A_{0}+A_{1}\left\{\begin{array}{c}
-\frac{a}{2 b}-\frac{\theta}{2 b} \tanh \left(\frac{1}{2} \theta z\right) \\
+\frac{\cosh (\theta z / 2)-(2 b / \theta) \sinh (\theta z / 2)}{C \cosh (\theta z / 2)-(2 b / \theta) \sinh (\theta z / 2)}
\end{array}\right\} \\
& +A_{2}\left\{\begin{array}{c}
\frac{a}{2 b}-\frac{\theta}{2 b} \tanh \left(\frac{1}{2} \theta z\right) \\
\operatorname{sech}(\theta z / 2)
\end{array}\right\}
\end{aligned}
$$

where $z=v x+y-(c+v) t$ and $C$ is an arbitrary constant of integration.

The solution (16) is a solitary wave solution in the form of a kink solution.

\section{Conservation Laws for (1)}

In this section we obtain conservation laws for the $(2+1)$ dimensional Boussinesq equation (1) using Ibragimov theorem [29], but first we give some definitions and notations which we will utilize later.

3.1. Fundamental Operators and Their Relationship. Let us consider a $k$ th-order system of PDEs of $n$ independent variables $x=\left(x^{1}, x^{2}, \ldots, x^{n}\right)$ and $m$ dependent variables $u=\left(u^{1}\right.$, $\left.u^{2}, \ldots, u^{m}\right)$, namely,

$$
E_{\alpha}\left(x, u, u_{(1)}, \ldots, u_{(k)}\right)=0, \quad \alpha=1, \ldots, m .
$$

Here $u_{(1)}, u_{(2)}, \ldots, u_{(k)}$ denote the collections of all first, second, ..., $k$ th-order partial derivatives; that is, $u_{i}^{\alpha}=D_{i}\left(u^{\alpha}\right)$, $u_{i j}^{\alpha}=D_{j} D_{i}\left(u^{\alpha}\right), \ldots$, respectively, with the total derivative operator with respect to $x^{i}$ defined by

$$
D_{i}=\frac{\partial}{\partial x^{i}}+u_{i}^{\alpha} \frac{\partial}{\partial u^{\alpha}}+u_{i j}^{\alpha} \frac{\partial}{\partial u_{j}^{\alpha}}+\cdots, \quad i=1, \ldots, n .
$$


The Euler-Lagrange operator, for each $\alpha$, is defined by

$$
\begin{array}{r}
\frac{\delta}{\delta u^{\alpha}}=\frac{\partial}{\partial u^{\alpha}}+\sum_{s \geq 1}(-1)^{s} D_{i_{1}} \cdots D_{i_{s}} \frac{\partial}{\partial u_{i_{1} i_{2} \cdots i_{s}}^{\alpha}}, \\
\alpha=1, \ldots, m,
\end{array}
$$

and the Lie-Bäcklund operator is given by

$$
X=\xi^{i} \frac{\partial}{\partial x^{i}}+\eta^{\alpha} \frac{\partial}{\partial u^{\alpha}}, \quad \xi^{i}, \eta^{\alpha} \in \mathscr{A},
$$

where $\mathscr{A}$ is the space of differential functions. The operator (27) can be written as

$$
X=\xi^{i} \frac{\partial}{\partial x^{i}}+\eta^{\alpha} \frac{\partial}{\partial u^{\alpha}}+\sum_{s \geq 1} \zeta_{i_{1} i_{2} \cdots i_{s}}^{\alpha} \frac{\partial}{\partial u_{i_{1} i_{2} \cdots i_{s}}^{\alpha}},
$$

where

$$
\begin{gathered}
\zeta_{i}^{\alpha}=D_{i}\left(W^{\alpha}\right)+\xi^{j} u_{i j}^{\alpha}, \\
\zeta_{i_{1} \cdots i_{s}}^{\alpha}=D_{i_{1}} \cdots D_{i_{s}}\left(W^{\alpha}\right)+\xi^{j} u_{j i_{1} \cdots i_{s}}^{\alpha}, \quad s>1 .
\end{gathered}
$$

Here $W^{\alpha}$ is the Lie characteristic function defined by

$$
W^{\alpha}=\eta^{\alpha}-\xi^{i} u_{j}^{\alpha}
$$

We can write the Lie-Bäcklund operator (28) in characteristic form as

$$
\begin{aligned}
X= & \xi^{i} D_{i}+W^{\alpha} \frac{\partial}{\partial u^{\alpha}} \\
& +\sum_{s \geq 1} D_{i_{1}} \cdots D_{i_{s}}\left(W^{\alpha}\right) \frac{\partial}{\partial u_{i_{1} i_{2} \cdots i_{s}}^{\alpha}} .
\end{aligned}
$$

The Noether operators associated with a Lie-Bäcklund symmetry operator $X$ are defined as

$$
\begin{aligned}
N^{i}= & \xi^{i}+W^{\alpha} \frac{\delta}{\delta u_{i}^{\alpha}} \\
& +\sum_{s \geq 1} D_{i_{1}} \cdots D_{i_{s}}\left(W^{\alpha}\right) \frac{\delta}{\delta u_{i i_{1} i_{2} \cdots i_{s}}^{\alpha}}, \quad i=1, \ldots, n,
\end{aligned}
$$

where the Euler-Lagrange operators with respect to derivatives of $u^{\alpha}$ are obtained from (26) by replacing $u^{\alpha}$ by the corresponding derivatives. For example,

$$
\begin{array}{r}
\frac{\delta}{\delta u_{i}^{\alpha}}=\frac{\partial}{\partial u_{i}^{\alpha}}+\sum_{s \geq 1}(-1)^{s} D_{j_{1}} \cdots D_{j_{s}} \frac{\partial}{\partial u_{i j_{1} j_{2} \cdots j_{s}}^{\alpha}}, \\
i=1, \ldots, n, \quad \alpha=1, \ldots, m
\end{array}
$$

and the Euler-Lagrange, Lie-Bäcklund, and Noether operators are connected by the operator identity

$$
X+D_{i}\left(\xi^{i}\right)=W^{\alpha} \frac{\delta}{\delta u^{\alpha}}+D_{i} N^{i}
$$

The $n$-tuple vector $T=\left(T^{1}, T^{2}, \ldots, T^{n}\right), T^{j} \in \mathscr{A}, j=1, \ldots, n$, is a conserved vector of (24) if $T^{i}$ satisfies

$$
\left.D_{i} T^{i}\right|_{(11)}=0,
$$

which defines a local conservation law of system (24).

The system of adjoint equations to (24) is defined by

$$
E_{\alpha}^{*}\left(x, u, v, \ldots, u_{(k)}, v_{(k)}\right)=0, \quad \alpha=1, \ldots, m
$$

where

$$
\begin{array}{r}
E_{\alpha}^{*}\left(x, u, v, \ldots, u_{(k)}, v_{(k)}\right)=\frac{\delta\left(v^{\beta} E_{\beta}\right)}{\delta u^{\alpha}}, \\
\alpha=1, \ldots, m, \quad v=v(x)
\end{array}
$$

and $v=\left(v^{1}, v^{2}, \ldots, v^{m}\right)$ are new dependent variables.

The system of (24) is known as self-adjoint if the substitution of $v=u$ into the system of adjoint equations (36) yields the same system (24).

Let us now assume the system of (24) admits the symmetry generator

$$
X=\xi^{i} \frac{\partial}{\partial x^{i}}+\eta^{\alpha} \frac{\partial}{\partial u^{\alpha}}
$$

Then the system of adjoint equations (36) admits the operator

$$
\begin{aligned}
& Y=\xi^{i} \frac{\partial}{\partial x^{i}}+\eta^{\alpha} \frac{\partial}{\partial u^{\alpha}}+\eta_{*}^{\alpha} \frac{\partial}{\partial v^{\alpha}}, \\
& \eta_{*}^{\alpha}=-\left[\lambda_{\beta}^{\alpha} v^{\beta}+v^{\alpha} D_{i}\left(\xi^{i}\right)\right],
\end{aligned}
$$

where the operator (39) is an extension of (38) to the variable $v^{\alpha}$ and the $\lambda_{\beta}^{\alpha}$ are obtainable from

$$
X\left(E_{\alpha}\right)=\lambda_{\alpha}^{\beta} E_{\beta}
$$

We now state the following theorem.

Theorem 1 (see [29]). Every Lie point, Lie-Bäcklund, and nonlocal symmetry (38) admitted by the system of (24) gives rise to a conservation law for the system consisting of (24) and the adjoint equation (36), where the components $T^{i}$ of the conserved vector $T=\left(T^{1}, \ldots, T^{n}\right)$ are determined by

$$
\begin{array}{r}
T^{i}=\xi^{i} L+W^{\alpha} \frac{\delta L}{\delta u_{i}^{\alpha}}+\sum_{s \geq 1} D_{i_{1}} \cdots D_{i_{s}}\left(W^{\alpha}\right) \frac{\delta L}{\delta u_{i i_{1} i_{2} \cdots i_{s}}^{\alpha}}, \\
i=1, \ldots, n,
\end{array}
$$

with Lagrangian given by

$$
L=v^{\alpha} E_{\alpha}\left(x, u, \ldots, u_{(k)}\right) .
$$


3.2. Construction of Conservation Laws for (1). In this subsection, we obtain conservation laws of $(2+1)$-dimensional Boussinesq equation

$$
u_{t t}-u_{x x}-u_{y y}-2 \alpha u_{x}^{2}-2 \alpha u u_{x x}-u_{x x x x}=0
$$

Recall that (43) admits the following five Lie point symmetry generators:

$$
\begin{gathered}
R_{1}=\frac{\partial}{\partial x}, \quad R_{2}=\frac{\partial}{\partial t}, \quad R_{3}=\frac{\partial}{\partial y}, \\
R_{4}=y \frac{\partial}{\partial t}+t \frac{\partial}{\partial y}, \\
R_{5}=-2 \alpha t \frac{\partial}{\partial t}-x \alpha \frac{\partial}{\partial x}-2 \alpha y \frac{\partial}{\partial y}+(1+2 \alpha u) \frac{\partial}{\partial u} .
\end{gathered}
$$

We now find five conserved vectors corresponding to each of these five Lie point symmetries.

The adjoint equation of (43), by invoking (37), is

$$
\begin{gathered}
E^{*}\left(t, x, u, v, \ldots, u_{x x x x}, v_{x x x x}\right) \\
=\frac{\delta}{\delta u}\left[v \left(u_{t t}-u_{x x}-u_{y y}-2 \alpha u_{x}^{2},\right.\right. \\
\left.\left.-2 \alpha u u_{x x}-u_{x x x x}\right)\right]=0,
\end{gathered}
$$

where $v=v(t, x, y)$ is a new dependent variable and (45) gives

$$
v_{t t}-v_{x x}-v_{y y}-2 \alpha u v_{x x}-v_{x x x x}=0 .
$$

It is obvious from the adjoint equation (46) that (43) is not self-adjoint. By recalling (42), we get the following Lagrangian for the system of (43) and (46):

$$
L=v\left(u_{t t}-u_{x x}-u_{y y}-2 \alpha u_{x}^{2}-2 \alpha u u_{x x}-u_{x x x x}\right) \text {. }
$$

(i) We first consider the Lie point symmetry generator $R_{1}=\partial / \partial x$. It can be verified from (39) that the operator $Y_{1}$ is the same as $R_{1}$ and the Lie characteristic function is $W=-u_{x}$. Thus, by using (41), the components $T^{i}, i=1,2,3$, of the conserved vector $T=\left(T^{1}, T^{2}, T^{3}\right)$ are given by

$$
\begin{gathered}
T^{1}=u_{x} v_{t}-v u_{t x}, \\
T^{2}=v u_{t t}-v u_{y y}-u_{x} v_{x}-2 \alpha u u_{x} v_{x} \\
-u_{x} v_{x x x}+v_{x x} u_{x x}-v_{x} u_{x x x} \\
T^{3}=-u_{x} v_{y}+v u_{x y} .
\end{gathered}
$$

Remark 2. The conserved vector $T$ contains the arbitrary solution $v$ of the adjoint equation (46) and hence gives an infinite number of conservation laws.

The same remark applies to all the following four cases.

(ii) Now for the second symmetry generator $R_{2}=\partial / \partial t$, we have $W=-u_{t}$. Hence, by invoking (41), the symmetry generator $R_{2}$ gives rise to the following components of the conserved vector:

$$
\begin{gathered}
T^{1}=-v u_{x x}-v u_{y y}-2 \alpha v u_{x}^{2}-2 \alpha u v u_{x x}-v u_{x x x x}+u_{t} v_{t}, \\
T^{2}=-u_{t} v_{x}+2 \alpha v u_{t} u_{x}-2 \alpha u u_{t} v_{x}-u_{t} v_{x x x} \\
+v u_{t x}+2 \alpha u v u_{t x}+v_{x x} u_{t x}-v_{x} u_{t x x}+v u_{t x x x} \\
T^{3}=-v_{y} u_{t}+v u_{t y} .
\end{gathered}
$$

(iii) The third symmetry generator, $R_{3}=\partial / \partial y$, gives $W=$ $-u_{y}$ and the corresponding components of the conserved vector are

$$
\begin{gathered}
T^{1}=v_{t} u_{y}-v u_{t y}, \\
T^{2}=-u_{y} v_{x}+2 \alpha v u_{y} u_{x}-2 \alpha u u_{y} v_{x}-u_{y} v_{x x x}+v u_{x y} \\
+2 \alpha u v u_{x y}+v_{x x} u_{x y}-v_{x} u_{x x y}+v u_{x x x y}, \\
T^{3}=v u_{t t}-v u_{x x}-2 \alpha v u_{x}^{2}-2 \alpha u v u_{x x}-v u_{x x x x}-u_{y} v_{y} .
\end{gathered}
$$

(iv) For the symmetry generator $R_{4}=y \partial / \partial t+t \partial / \partial y$ the components of the conserved vector, as before, are given by

$$
\begin{aligned}
T^{1}= & -y v u_{x x}-y v u_{y y}-2 \alpha y v u_{x}^{2} \\
& -2 \alpha y u v u_{x x}-y v u_{x x x x}+y u_{t} v_{t} \\
& +t u_{y} v_{t}-v u_{y}-t v u_{t y}, \\
T^{2}= & -y u_{t} v_{x}+2 \alpha y v u_{t} u_{x}-2 \alpha y u u_{t} v_{x} \\
& -y u_{t} v_{x x x}-t v_{x} u_{y}+2 \alpha t v u_{y} u_{x}-2 \alpha t u v_{x} u_{y} \\
& -t u_{y} v_{x x x}+y v u_{t x}+2 \alpha y u v u_{t x}+y u_{t x} v_{x x} \\
& +t v u_{x y}+2 \alpha t u v u_{x y}+t u_{x y} v_{x x}-y v_{x} u_{t x x} \\
& -t v_{x} u_{x x y}+y v u_{t x x x}+t v u_{x x x y}, \\
T^{3}= & t v u_{t t}-t v u_{x x}-2 \alpha t v u_{x}^{2} \\
& -2 \alpha t u v u_{x x}-t v u_{x x x x}-y v_{y} u_{t} \\
& -t v_{y} u_{y}+v u_{t}+y v u_{t y} .
\end{aligned}
$$

(v) Finally, for the symmetry generator

$$
R_{5}=-2 \alpha t \frac{\partial}{\partial t}-x \alpha \frac{\partial}{\partial x}-2 \alpha y \frac{\partial}{\partial y}+(1+2 \alpha u) \frac{\partial}{\partial u}
$$

the value of $Y_{5}$ is different from $R_{5}$ and is given by

$$
Y_{5}=-2 \alpha t \frac{\partial}{\partial t}-x \alpha \frac{\partial}{\partial x}-2 \alpha y \frac{\partial}{\partial y}+(1+2 \alpha u) \frac{\partial}{\partial u}-v \alpha \frac{\partial}{\partial v}
$$


In this case the Lie characteristic function is $W=1+2 \alpha u+$ $2 \alpha t u_{t}+x \alpha u_{x}+2 \alpha y u_{y}$. So using (41), one can obtain the conserved vector $T$ whose components are given by

$$
\begin{aligned}
& T^{1}=2 \alpha t v u_{x x}+2 \alpha t v u_{y y}+4 \alpha^{2} t v u_{x}^{2} \\
& +4 \alpha^{2} t v u u_{x x}+2 \alpha t v u_{x x x x}-v_{t} \\
& -2 \alpha u v_{t}-2 \alpha t u_{t} v_{t}-x \alpha u_{x} v_{t}-2 \alpha y u_{y} v_{t} \\
& +4 \alpha v u_{t}+x \alpha v u_{t x}+2 \alpha y v u_{t y}, \\
& T^{2}=-x \alpha v u_{t t}+x \alpha v u_{y y}+2 \alpha x u v u_{x x} \\
& +v_{x}+4 \alpha u v_{x}+v_{x x x}+4 \alpha^{2} u^{2} v_{x} \\
& +2 \alpha u v_{x x x}-8 \alpha t v u_{x} u_{t}+2 \alpha t u_{t} v_{x} \\
& +4 \alpha^{2} t v u_{t} u_{x}+4 \alpha^{2} t u u_{t} v_{x}+2 \alpha t u_{t} v_{x x x} \\
& +x \alpha u_{x} v_{x}+2 \alpha^{2} x u u_{x} v_{x}+x \alpha u_{x} v_{x x x} \\
& -8 \alpha^{2} y v u_{x} u_{y}+2 \alpha y u_{y} v_{x}+4 \alpha^{2} y v u_{x} u_{y} \\
& +4 \alpha^{2} y u u_{y} v_{x}+2 \alpha y u_{y} v_{x x x}-2 \alpha t v u_{t x} \\
& -5 \alpha v u_{x}-2 \alpha y v u_{x y}-4 \alpha^{2} t u v u_{t x} \\
& -10 \alpha^{2} u v u_{x}-2 \alpha^{2} x u v u_{x x}-4 \alpha^{2} y u v u_{x y} \\
& -3 \alpha u_{x} v_{x x}-2 \alpha t u_{t x} v_{x x}-x \alpha u_{x x} v_{x x} \\
& -2 \alpha y u_{x y} v_{x x}+2 \alpha t v_{x} u_{t x x}+4 \alpha v_{x} u_{x x} \\
& +x \alpha v_{x} u_{x x x}+2 \alpha y v_{x} u_{x x y}-2 \alpha t v u_{t x x x} \\
& -5 \alpha v u_{x x x}-2 \alpha y v u_{x x x y}, \\
& T^{3}=-2 \alpha y v u_{t t}+2 \alpha y v u_{x x}+4 \alpha^{2} y v u_{x}^{2} \\
& +4 \alpha^{2} y v u u_{x x}+2 \alpha y v u_{x x x x}+v_{y} \\
& +2 \alpha u v_{y}+2 \alpha t u_{t} v_{y}+x \alpha u_{x} v_{y}+2 \alpha y u_{y} v_{y} \\
& -4 \alpha v u_{y}-x \alpha v u_{x y}-2 \alpha t v u_{t y} .
\end{aligned}
$$

\section{Conclusions}

In this paper, for the first time, Lie symmetries as well as the simplest equation method were used to obtain exact solutions of the $(2+1)$-dimensional Boussinesq equation (1). The solutions obtained were solitary waves and nontopological soliton. It is obvious from the analysis we conducted that the $(2+1)$-dimensional equation gives rise to a variety of solitary wave solutions that ranges from kink to soliton solutions. The obtained kink and soliton solutions are regular solitons given in the form of hyperbolic tan or the $\operatorname{sech}^{2}$ form. Moreover, the conservation laws for the $(2+1)$-dimensional Boussinesq equation were also derived by using the new conservation theorem due to Ibragimov [29].

\section{References}

[1] C. Gu, H. Hu, and Z. Zhou, Darboux Transformations in Integrable Systems, vol. 26 of Mathematical Physics Studies, Springer, Dordrecht, Netherlands, 2005.

[2] M. J. Ablowitz and P. A. Clarkson, Solitons, Nonlinear Evolution Equations and Inverse Scattering, vol. 149 of London Mathematical Society Lecture Note Series, Cambridge University Press, Cambridge, UK, 1991.

[3] R. Hirota, The Direct Method in Soliton Theory, vol. 155 of Cambridge Tracts in Mathematics, Cambridge University Press, Cambridge, UK, 2004.

[4] S. Liu, Z. Fu, S. Liu, and Q. Zhao, "Jacobi elliptic function expansion method and periodic wave solutions of nonlinear wave equations," Physics Letters A, vol. 289, no. 1-2, pp. 69-74, 2001.

[5] A.-M. Wazwaz, "The tanh and the sine-cosine methods for compact and noncompact solutions of the nonlinear KleinGordon equation," Applied Mathematics and Computation, vol. 167, no. 2, pp. 1179-1195, 2005.

[6] Sirendaoreji and S. Jiong, "Auxiliary equation method for solving nonlinear partial differential equations," Physics Letters A, vol. 309, no. 5-6, pp. 387-396, 2003.

[7] G. W. Bluman and S. Kumei, Symmetries and Differential Equations, vol. 81 of Applied Mathematical Sciences, Springer, New York, NY, USA, 1989.

[8] P. J. Olver, Applications of Lie Groups to Differential Equations, vol. 107 of Graduate Texts in Mathematics, Springer, New York, NY, USA, 2nd edition, 1993.

[9] N. H. Ibragimov, CRC Handbook of Lie Group Analysis of Differential Equations, vol. 1-3, CRC Press, Boca Raton, Fla, USA, 1994-1996.

[10] C. M. Khalique and A. R. Adem, "Exact solutions of a generalized (3+1)-dimensional Kadomtsev-Petviashvili equation using Lie symmetry analysis," Applied Mathematics and Computation, vol. 216, no. 10, pp. 2849-2854, 2010.

[11] K. R. Adem and C. M. Khalique, "Exact solutions and conservation laws of a $(2+1)$-dimensional nonlinear KP-BBM equation," Abstract and Applied Analysis, vol. 2013, Article ID 791863, 5 pages, 2013.

[12] M. Wang and Y. Zhou, "The periodic wave solutions for the Klein-Gordon-Schrödinger equations," Physics Letters A, vol. 318, no. 1-2, pp. 84-92, 2003.

[13] J.-H. He and X.-H. Wu, "Exp-function method for nonlinear wave equations," Chaos, Solitons \& Fractals, vol. 30, no. 3, pp. 700-708, 2006.

[14] S. Lai, Y. Wu, and Y. Zhou, "Some physical structures for the $(2+1)$-dimensional Boussinesq water equation with positive and negative exponents," Computers \& Mathematics with Applications, vol. 56, no. 2, pp. 339-345, 2008.

[15] Y. Chen, Z. Yan, and H. Zhang, "New explicit solitary wave solutions for $(2+1)$-dimensional Boussinesq equation and $(3+$ 1)-dimensional KP equation," Physics Letters A, vol. 307, no. 2-3, pp. 107-113, 2003.

[16] G. Imed and B. Abderrahmen, "Numerical solution of the $(2+$ 1)-dimensional Boussinesq equation with initial condition by homotopy perturbation method," Applied Mathematical Sciences, vol. 6, no. 117-120, pp. 5993-6002, 2012.

[17] C. Liu and Z. Dai, "Exact periodic solitary wave solutions for the $(2+1)$-dimensional Boussinesq equation," Journal of Mathematical Analysis and Applications, vol. 367, no. 2, pp. 444-450, 2010. 
[18] A.-M. Wazwaz, "Non-integrable variants of Boussinesq equation with two solitons," Applied Mathematics and Computation, vol. 217, no. 2, pp. 820-825, 2010.

[19] P. D. Lax, "Integrals of nonlinear equations of evolution and solitary waves," Communications on Pure and Applied Mathematics, vol. 21, pp. 467-490, 1968.

[20] T. B. Benjamin, "The stability of solitary waves," Proceedings of the Royal Society A, vol. 328, pp. 153-183, 1972.

[21] R. J. Knops and C. A. Stuart, "Quasiconvexity and uniqueness of equilibrium solutions in nonlinear elasticity," Archive for Rational Mechanics and Analysis, vol. 86, no. 3, pp. 233-249, 1984.

[22] R. J. LeVeque, Numerical Methods for Conservation Laws, Lectures in Mathematics, Birkhäuser, Basel, Switzerland, 2nd edition, 1992.

[23] E. Godlewski and P.-A. Raviart, Numerical Approximation of Hyperbolic Systems of Conservation Laws, vol. 118 of Applied Mathematical Sciences, Springer, Berlin, Germany, 1996.

[24] A. Sjöberg, "Double reduction of PDEs from the association of symmetries with conservation laws with applications," Applied Mathematics and Computation, vol. 184, no. 2, pp. 608-616, 2007.

[25] A. H. Bokhari, A. Y. Al-Dweik, A. H. Kara, F. M. Mahomed, and F. D. Zaman, "Double reduction of a nonlinear $(2+1)$ wave equation via conservation laws," Communications in Nonlinear Science and Numerical Simulation, vol. 16, no. 3, pp. 1244-1253, 2011.

[26] G. L. Caraffini and M. Galvani, "Symmetries and exact solutions via conservation laws for some partial differential equations of mathematical physics," Applied Mathematics and Computation, vol. 219, no. 4, pp. 1474-1484, 2012.

[27] N. A. Kudryashov, "Simplest equation method to look for exact solutions of nonlinear differential equations," Chaos, Solitons \& Fractals, vol. 24, no. 5, pp. 1217-1231, 2005.

[28] N. K. Vitanov, "Application of simplest equations of Bernoulli and Riccati kind for obtaining exact traveling-wave solutions for a class of PDEs with polynomial nonlinearity," Communications in Nonlinear Science and Numerical Simulation, vol. 15, no. 8, pp. 2050-2060, 2010.

[29] N. H. Ibragimov, "A new conservation theorem," Journal of Mathematical Analysis and Applications, vol. 333, no. 1, pp. 311328, 2007. 


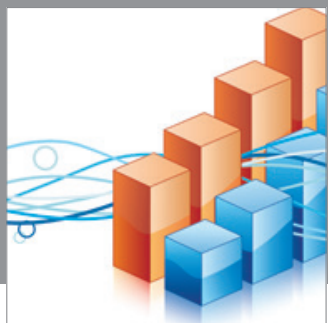

Advances in

Operations Research

mansans

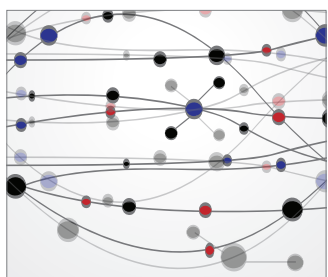

The Scientific World Journal
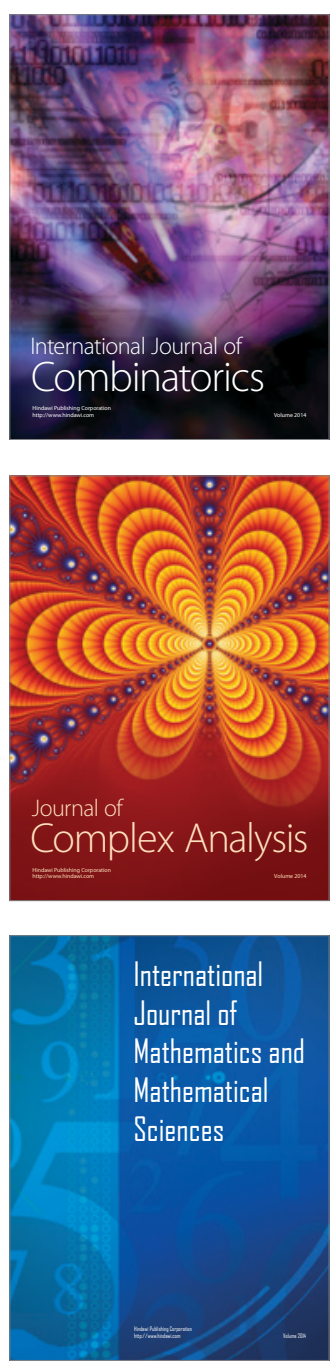
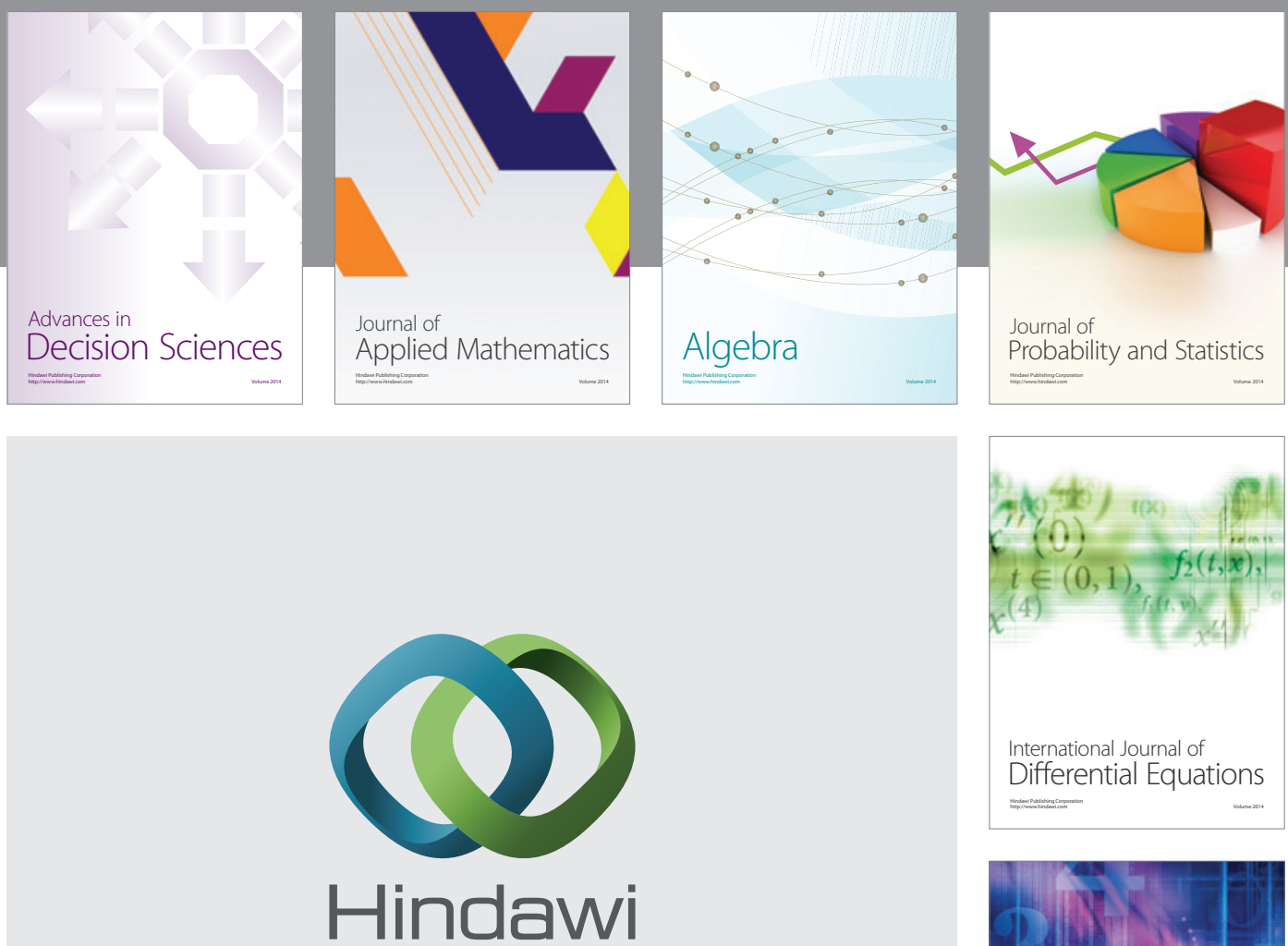

Submit your manuscripts at http://www.hindawi.com
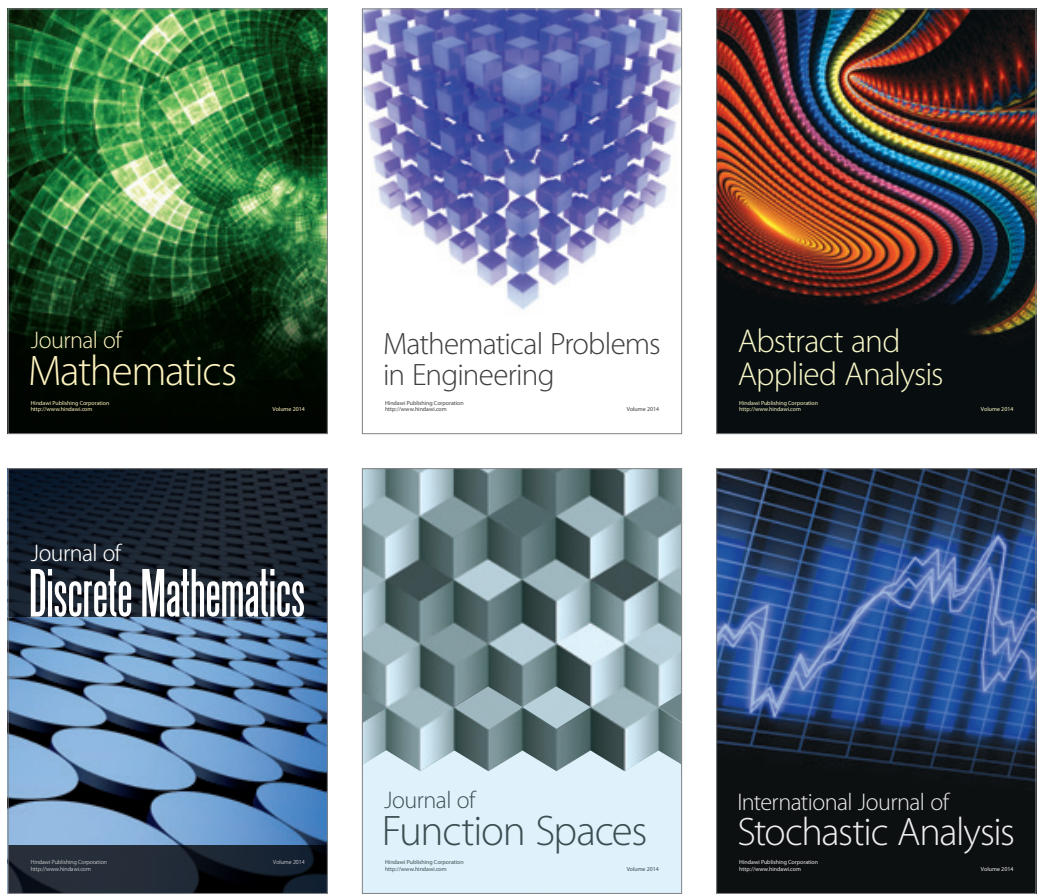

Journal of

Function Spaces

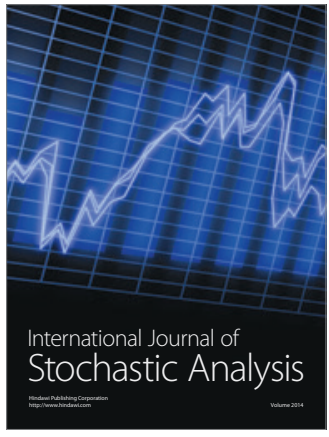

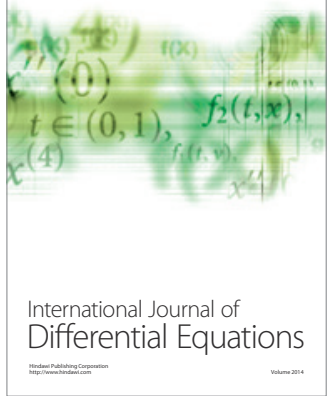
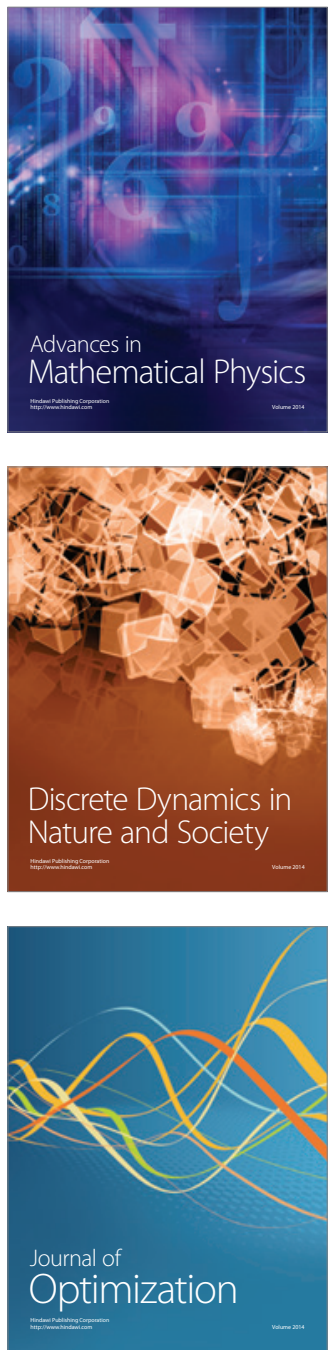